\title{
HIGHLIGHTS
}

OBESITY

\section{Maternal obesity may predispose offspring to IBD}

Results from a new study in sheep by researchers at the Center for the Study of Fetal Programming at the University of Wyoming, USA, show that maternal obesity may predispose offspring to developing IBD.

Currently, the incidence of obesity in American women, including pregnant women, is increasing rapidly. The incidence of IBD is also increasing, especially in children. "Accumulating convincing evidence indicates that maternal obesity may adversely affect fetal growth and development, which can result in harmful persistent effects in offspring," says Mei-Jun Zhu, corresponding author of the study. "We sought to determine if there is a link between maternal obesity and the occurrence of IBD in offspring."

The researchers used sheep in their investigations because the temporal pattern of fetal development and the ratio of fetal to maternal weight is similar to that of humans. Nonpregnant ewes were assigned to a control diet or an obesogenic diet from 60 days prior to conception until day 135 of gestation. Tissue from the large intestine of fetuses (at 135 days after conception) and from offspring (at $22.5 \pm 0.5$ months of age) were sampled and analyzed.

"The most significant finding is that maternal obesity leads to the production of inflammatory cytokines and enhanced inflammatory signaling in the fetal large intestine by late gestation," reports Zhu. "This inflammation persists in the offspring gut postnatally, which may predispose these offspring to IBD.” In particular, Zhu and colleagues found that maternal obesity was associated with increased messenger RNA (mRNA) expression of Toll-like receptor (TLR)2 and TLR4 in fetuses and offspring; TLRs have an important role in inducing inflammation.

Maternal obesity was also associated with increased expression of the inflammatory cytokines tumor necrosis factor (TNF), interleukin (IL)-1 $\alpha$, IL-1 $\beta$, IL-8, IL-6 and monocyte chemotactic protein 1 (MCP1). These findings are important as IL-6 is necessary for the differentiation of $\mathrm{T}_{\mathrm{H}} 17$ cells, which are

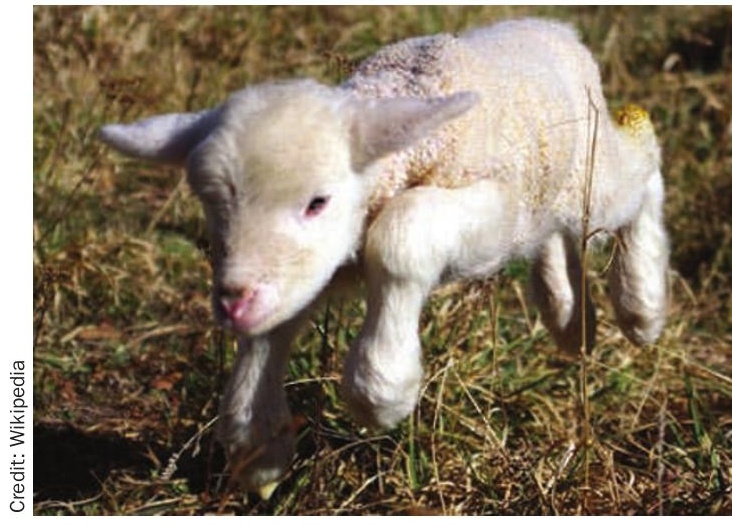

associated with the development of IBD. The mRNA expression of macrophage markers CD11b, CD14 and CD68 was also increased in the fetuses and offspring of obese mothers.

"We want to define the underlying mechanisms whereby maternal obesity leads to persistent inflammation in the offspring gut," concludes Zhu. "In addition, we want to develop clinically relevant methods to prevent this in utero change from occurring in obese pregnancies."

\section{Isobel Franks}

Original article Yan, X. et al. Maternal obesity induces sustained inflammation in both fetal and offspring large intestine of sheep. Inflamm. Bowel Dis. doi:10.1002/ ibd.21539 